\title{
Fecundidad y desarrollo gonadal de la "boga" Leporinus obtusidens (Pisces, Anostomidae) en la represa Yacyretá, Argentina
}

\author{
Flores Quintana, C.; Arbués, R.; Sánchez, S.; González, A.; Fontana, D. \\ Instituto de Ictiología del Nordeste (INICNE), Facultad de Ciencias Veterinarias, UNNE, \\ Sargento Cabral 2139, Corrientes (3400), Argentina. Tel/fax: 03783-425753, \\ E-mail: carolina@vet.unne.edu.ar.
}

\begin{abstract}
Resumen
Flores Quintana, C.; Arbués, R.; Sánchez, S.; González, A.; Fontana, D.: Fecundidad y desarrollo gonadal de la "boga" Leporinus obtusidens (Pisces, Anostomidae) en la represa Yacyretá, Argentina. Rev. vet. 21: 1, 48-54, 2010. En el presente trabajo se exponen los resultados obtenidos durante cinco años consecutivos de muestreos de boga Leporinus obtusidens en dos puntos del río Paraná, Argentina, considerando a uno de ellos como zona de impacto de la represa Yaciretá y otro sitio, lejano a la misma, como zona control. Se analiza la relación gonadosomática, las fecundidades absoluta y relativa y se describen las características morfológicas de los ovocitos en desarrollo. A pesar de las numerosas modificaciones que impone la presencia de una represa en las características de un río, los resultados sugieren que la boga no muestra repercusiones negativas en varios aspectos relacionados a la biología reproductiva en respuesta a las condiciones ambientales cambiantes y a niveles hídricos que fluctúan rápidamente.
\end{abstract}

Palabras clave: pez, Leporinus obtusidens, relación gonadosomática, ovocitos.

\begin{abstract}
Flores Quintana, C.; Arbués, R.; Sánchez, S.; González, A.; Fontana, D.: Gonadal development and fertility of the "boga" (Leporinus obtusidens) in Yacyretá dam (Argentina). Rev. vet. 21: 1, 48-54, 2010. The aim of this work is to present the results of a five years survey on gonadosomatic index as well as absolute and relative fecundity and morphological characteristics of developing oocytes of Leporinus obtusidens ("boga") from two areas of the Parana River, Argentina, one located in the proximity of Yaciretá dam and considered of great environmental impact, and the other far away from the dam (control). Results indicate that, despite the numerous environmental modifications due to the presence of the dam such as fluctuating water levels, negative impact was not observed over reproduction of $L$. obtusidens.
\end{abstract}

Key words: fish, Leporinus obtusidens, gonadosomatic relationship, oocytes.

\section{INTRODUCCIÓN}

En las últimas décadas, la construcción de numerosas represas en las principales cuencas hidrográficas de América del Sur trajo como consecuencia importantes modificaciones en el flujo y en el tiempo de residencia del agua, factores que repercutieron en sus características físicas, químicas y biológicas. Las represas imponen restricciones en las vías migratorias y causan modificaciones en el hábitat. El nivel hídrico, controlado con abertura y cierres de vertederos, ocasiona marcados cambios en el caudal que no ocurren naturalmente, afectando la biología de las especies ícticas ${ }^{11}$.

Los ovarios de los peces teleósteos son generalmente pares y alargados. El peso, volumen y la colo-

Recibido: 27 abril 2010 / Aceptado: 18 junio 2010 ración sufren intensas modificaciones durante el ciclo reproductivo, que comprende dos fases diferentes: la del desarrollo gonadal y la del desove, cada una con un mecanismo de control diferente. Durante el desarrollo gonadal el crecimiento de la gónada va desde el estado de reposo, que comprende menos del $0,1 \%$ del peso corporal (relación gonadosomática RGS), hasta el estado de maduración completa, cuando puede alcanzar una RGS del 20 al 30\%. El crecimiento requiere una significativa movilización de energía y se desenvuelve en un período de tiempo que demanda varios meses, se inicia con un fotoperíodo específico, temperatura y condiciones ambientales determinadas que son fehacientes indicadores de estacionalidad. Diferentes tipos de estrés repercuten en el proceso reproductivo y afectan la calidad de las gametas ${ }^{12,15}$. 
Si bien la presencia de represas en los grandes cuerpos de agua modifica el ecosistema e impacta sobre el recurso pesquero reduciendo o eliminando especies migradoras ${ }^{1}$, se sabe que los peces desarrollan diversas estrategias reproductivas para adaptarse a las condiciones ambientales cambiantes y a niveles hídricos que fluctúan rápidamente. El éxito de esa adaptación, que va a determinar la permanencia o no de las especies en el tiempo, puede ser monitoreada con estudios de fecundidad y desarrollo gonadal, variables que se consideran prioritarias para establecer eventuales medidas de manejo.

El objetivo de este trabajo fue caracterizar los ovarios de Leporinus obtusidens, especie que mostró marcado aumento de biomasa desde $1998^{5}$. La caracterización se realizó considerando los cambios cíclicos macro y microscópicos registrados durante cinco años consecutivos de muestreo, detectando posibles diferencias entre un sitio cercano y otro lejano a la represa Yacyretá (Corrientes, Argentina).

\section{MATERIAL Y MÉTODOS}

Se analizaron muestras de ovarios de Leporinus obtusidens capturados en muestreos mensuales desde el año 2004 al 2009, en dos sitios sobre la margen izquierda del río Paraná en la Provincia de Corrientes, Argentina: Ita Ibaté, considerado como control e Ituzaingó considerado sitio de impacto. Cada ciclo de muestreo analizado se inició en julio y se extendió a junio del año siguiente.

Los peces se capturaron con una batería de redes enmalladoras de nylon monofilamento, con aberturas de malla de $4 ; 5 ; 6 ; 7 ; 8 ; 12 ; 14 ; 16$ y $20 \mathrm{~cm}$ de nudo a nudo y otras artes de pesca complementarias como cañas con reel, tarrafas y redes de arrastre. De cada ejemplar capturado se registró el peso y la longitud estándar así como el peso de las gónadas Las gónadas fueron retiradas y pesadas. Macroscópicamente se examinó el color, transparencia, vascularización, flaccidez, visualización de ovocitos y espesor de la cápsula, y se determinó el estado de desarrollo ovárico utilizando la clasificación: virginal, inicio de maduración, maduración, desove, post-desove y reposo.

De cada gónada se tomó una muestra de alrededor de $1 \mathrm{~g}$, la que se fijó en solución de Bouin para el análisis histológico. Adicionalmente, de aquellos ejemplares maduros y próximos a desovar se fijó una segunda muestra en formol al 10\% para realizar el recuento de ovocitos maduros en cámara Sedgwick-Rafter bajo microscopio estereoscópico, a partir de una submuestra de peso conocido del material fijado.

La fecundidad total se refirió al peso fresco de la gónada obtenido en campo. El indice de fecundidad absoluta se calculó con la fórmula convencional (número total de ovocitos $=$ peso de la gónada $\mathrm{x}$ número de ovocitos por gramo de gónada). Además se calculó el índice de fecundidad relativa utilizando la fórmula: total de ovocitos maduros o en maduración / peso del pez en kilogramos. La relación gonadosomática (RGS) fue calculada con la fórmula RGS (\%) = peso total del ovario en gramos / peso total del pez en gramos x 100 .

Para el análisis histológico, las muestras fijadas en Bouin fueron posteriormente deshidratadas e incluidas en parafina. Los cortes histológicos coloreados con hematoxilina-eosina se analizaron con microscopio óptico para recuento de ovocitos e identificación de los estadios. Los ovocitos se clasificaron de acuerdo a su desarrollo en 6 fases según la metodología habitualmente utilizada ${ }^{4,18}$. Se consideraron como ovocitos en fase I o nucleolocromatínicos, a aquellos ovocitos más pequeños. Los ovocitos en fase II o perinucleolares fueron aquellos que presentaron núcleo central con nucleolos periféricos, citoplasma de contorno anguloso, con diferente afinidad tintorial dependiendo de su tamaño, con presencia o no de corpúsculos de Balbiani, estructura basófila, redondeada y homogénea. Ovocitos en fase III o alvéolo-corticales, fueron aquellas células que evidenciaron disposición periférica de los alvéolos corticales y membrana pelúcida, además de estar rodeadas por células foliculares. Los ovocitos en fase IV o vitelogénicos se identificaron por su mayor tamaño, envoltura folicular, granulación periférica y gránulos de vitelo de diferente tamaño. Los ovocitos atrésicos fueron células de núcleo excéntrico, sin contornos nítidos y nucleolos todavía evidentes.

Los índices de fecundidad absoluta y relativa de las hembras maduras de boga capturadas a lo largo de todo el estudio se examinaron estadísticamente mediante análisis de la covarianza (ANCOVA), considerando para ambas variables dependientes el efecto del sitio de muestreo y del ciclo anual. En el caso de la fecundidad relativa se utilizó como covariable la RGS, mientras que en el análisis de la fecundidad absoluta la covariable fue el peso total de las hembras. Las comparaciones a posteriori entre ciclos se realizaron mediante el test de Tuckey $(\mathrm{p}<0,05)$. Los cálculos estadísticos se realizaron con el auxilio del paquete GLM del programa Statistica 6.0.

\section{RESULTADOS Y DISCUSIÓN}

En las Figuras 1 y 2 se presentan las modificaciones de la relación gonadosomática durante todos los ciclos de muestreos en ambos sitios geográficos considerados.

La relación gonadosomática, frecuentemente usada para identificar los estadios de maduración gonadal ${ }^{13}$, ${ }^{14}$, refleja las alteraciones estructurales de las gónadas permitiendo inferir la condición reproductiva de los peces. En ambos sitios se detectó que la actividad reproductiva estuvo presente en un único período del año (noviembre-enero) con un marcado acmé en diciembre. El periodo de reproducción finalizó en febrero, observándose descenso marcado de la RGS

El análisis comparativo con los resultados de investigaciones anteriores ${ }^{4}$ permite notar la poca variación de los promedios de RGS obtenidos en el presente es- 
Tabla 1. Fecundidad relativa (óvulos maduros o en maduración por $\mathrm{g}$ de peso) de los distintos ciclos en ambos sitios de muestreo.

\begin{tabular}{lcccccc}
\hline \multirow{2}{*}{ ciclo } & \multicolumn{3}{c}{ control (Itá Ibaté) } & \multicolumn{3}{c}{ impacto (Ituzaingó) } \\
\cline { 2 - 7 } & $\mathrm{n}$ & promedio & máximo & $\mathrm{n}$ & promedio & máximo \\
\hline $2004-2005$ & 3 & 319,96 & 473,29 & 6 & 322,53 & 622,40 \\
$2005-2006$ & 16 & 392,44 & 546,62 & 11 & 419,68 & 583,03 \\
$2006-2007$ & 3 & 307,27 & 476,64 & 8 & 592,70 & 768,21 \\
$2007-2008$ & - & - & - & 9 & 395,28 & 684,82 \\
$2008-2009$ & 1 & 348,34 & 348,34 & 7 & 250,97 & 409,71 \\
\hline
\end{tabular}

Tabla 2. Fecundidades absoluta y relativa según período considerado y sitio geográfico.

\begin{tabular}{|c|c|c|c|c|c|c|}
\hline \multicolumn{3}{|c|}{ ANCOVA - fecundidad absoluta } & ciclo & LSM & sitio & LSM \\
\hline \multirow{5}{*}{$\begin{array}{l}\text { sitio } \\
\text { ciclo } \\
\text { covariab.(peso) }\end{array}$} & $\mathrm{F}$ & $\mathrm{p}$ & $04-05$ & $76003,8 \mathrm{~b}$ & Itá & \multirow{2}{*}{$147203,9 \mathrm{a}$} \\
\hline & 1,989182 & 0,16487 & $05-06$ & $151223,9 \mathrm{~b}$ & Ibaté & \\
\hline & 3,769356 & 0,00957 & $06-07$ & $197109,3 \mathrm{ab}$ & \multirow{3}{*}{ Ituzaingó } & \multirow{3}{*}{$213076,6 \mathrm{a}$} \\
\hline & 9,810741 & 0,00295 & $07-08$ & $92388,5 \mathrm{~b}$ & & \\
\hline & & & 08-09 & 290540,8 a & & \\
\hline \multicolumn{3}{|c|}{ ANCOVA - fecundidad relativa } & ciclo & LSM & sitio & LSM \\
\hline \multirow{5}{*}{$\begin{array}{l}\text { sitio } \\
\text { ciclo } \\
\text { covariab.(RGS) }\end{array}$} & $\mathrm{F}$ & $\mathrm{p}$ & $04-05$ & $349,59 \mathrm{~b}$ & Itá & \multirow{2}{*}{$380,35 \mathrm{a}$} \\
\hline & 0,0118 & 0,91385 & $05-06$ & $422,26 \mathrm{a}$ & Ibaté & \\
\hline & 3,9050 & 0,00797 & $06-07$ & $431,83 \mathrm{a}$ & \multirow{3}{*}{ Ituzaingó } & \multirow{3}{*}{$383,22 \mathrm{a}$} \\
\hline & 237,9030 & 0,00000 & $07-08$ & $392,85 \mathrm{ab}$ & & \\
\hline & & & 08-09 & $312,40 \mathrm{~b}$ & & \\
\hline
\end{tabular}

LSM: medias de mínimos cuadrados. Letras diferentes indican diferencias significativas en el test de Tuckey $(\mathrm{p}<0,05)$.

tudio. Sin embargo, los valores anteriores fueron superiores en Ita Ibaté. A partir del ciclo 2005-2006 se presentó una tendencia a la igualdad entre ambos sitios, que posteriormente se invirtió registrándose valores mayores en Ituzaingó, circunstancia que sugiere escaso impacto de la represa en la maduración gonadal.

El ciclo 2004-2005 mostró el promedio más alto del sitio control, en coincidencia con niveles hidrométricos muy bajos ${ }^{6}$. Se ha reportado que los períodos de sequía obligan a las especies a un esfuerzo reproductivo, ya que habría mayor mortalidad en las fases iniciales de desarrollo por la menor accesibilidad a los refugios, mayor predación y menor disponibilidad de

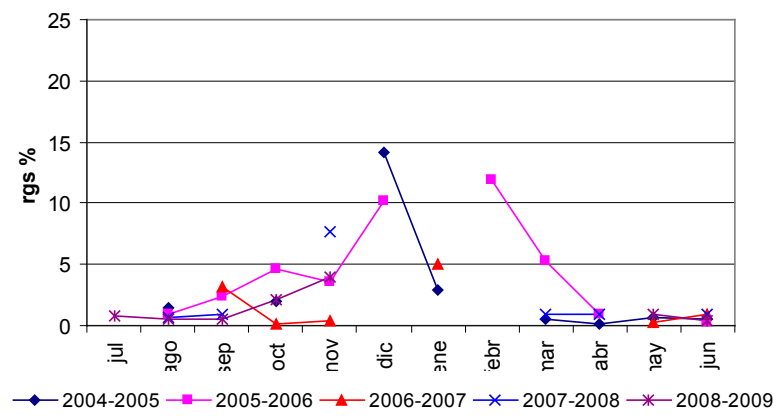

Figura 1. Relación gonadosomática (rgs) de Leporinus obtusidens desde julio de 2004 a junio de 2009 en la localidad de Ita Ibaté. alimento ${ }^{18}$. En Ituzaingó se destacan alteraciones diarias más pronunciadas del nivel hidrométrico, con característica ciclicidad semanal en los caudales, que se va atenuando aguas abajo ${ }^{5}$. Esta variabilidad hidrológica en cercanías de la represa podría ser favorable a especies generalistas, entre las que se encuentra la boga.

La fecundidad de los peces se puede definir desde el punto de vista absoluto (número total ovocitos que completan su desarrollo y que serán eliminados en el desove) o relativo (número ovulado por unidad de peso corporal). Si bien el número total es privativo para la especie, está relacionado con el tamaño del huevo y de la cavidad celómica que aloja las gónadas, variaciones genéticas, edad y tamaño corporal, entre otros factores. En el presente estudio, la fecundidad absoluta presentó variaciones entre sitios (Figuras 3 y 4) así como entre ciclos anuales, además de una relación positiva con el mayor peso de las hembras.

En la Tabla 1 se muestran los promedios y rangos máximos de fecundidad relativa de cada año, en los dos sitios geográficos estudiados. En la Tabla 2 se exponen los resultados del análisis de la covarianza realizado para analizar las variaciones de las fecundidades absoluta y relativa.

El análisis de la fecundidad absoluta utilizando el peso de las hembras como covariable solo permitió detectar diferencias estadísticamente significativas entre ciclos $(\mathrm{p}<0,05)$, al tiempo que se aprecia que el efecto de la covariable también resultó significativo, no existiendo diferencias estadísticas entre sitios de muestreo $(p>0,05)$.

En estudios anteriores ya se advirtió que los ejemplares de mayor porte de Leporinus obtusidens tendían

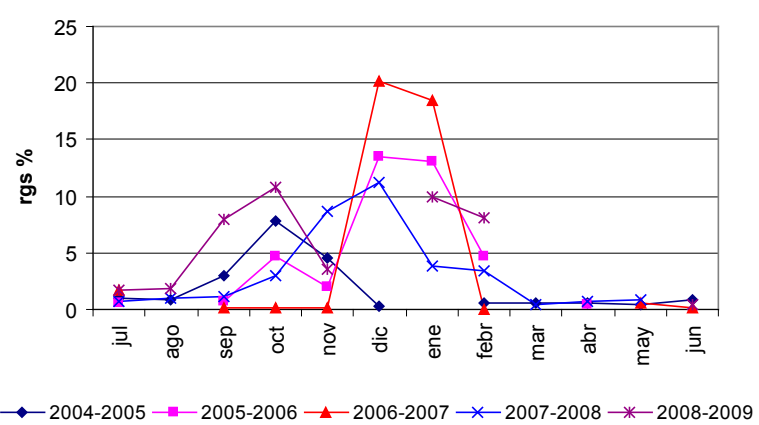

Figura 2. Relacion gonadosomática (rgs) de Leporinus obtusidens de julio 2004 a junio 2009 en la localidad de Ituzaingó. 
a aumentar su frecuencia relativa en cercanías de Yacyretá, donde la especie se caracterizó por un marcado aumento de biomasa, fecundidad y condición gonadosomática desde $1998^{5}$. Tal aumento fue atribuido a las condiciones favorables de alimentación ligadas a la presencia del molusco invasor Limnoperna fortunei hallado casi con exclusividad en contenidos estomacales de la boga. De manera similar, en cercanías de Itaipú (Paraguay) se observó que Leporinus obtusidens está lejos de desaparecer por el impacto de la represa, a diferencia de otras especies migradoras 1 . Asimismo, Leporinus friderici mostró una buena capacidad de adaptación en la represa de Lobo en Brasil ${ }^{2}$.

Los resultados obtenidos al analizar la fecundidad relativa fueron similares a los encontrados para la fecundidad absoluta (Tabla 2), ya que no se detectaron diferencias significativas entre sitios de muestreo $(\mathrm{p}>0,05)$ pero sí entre ciclos anuales $(\mathrm{p}<0,05)$. En relación a la covariable, la RGS de las hembras $(\mathrm{p}<0,05)$, se incrementó conforme al avance del proceso de maduración gonadal.

La proporción de sexos fue equilibrada en el sitio de impacto mientras que en el sitio control predominaron los machos (Tabla 3). Si se considera que la proporción por sexos refleja los desplazamientos con fines reproductivos ${ }^{18}$ podrían suponerse condiciones más favorables en Ituzaingó.

En años anteriores siempre hubo más hembras que machos en Ituzaingó y la distribución equilibrada estaba en Ita Ibaté ${ }^{4}$. Además de las migraciones reproductivas, algunos trabajos señalan que la mortalidad es uno de los factores que pueden actuar de modo diferencial sobre machos y hembras, determinando el predominio de un sexo. Sin embargo,

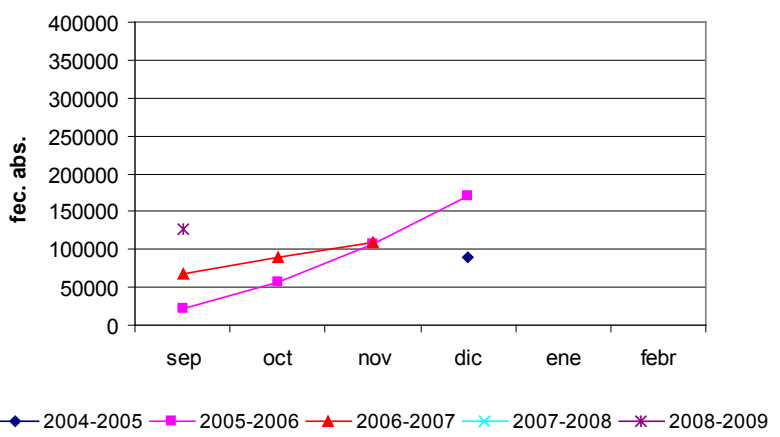

Figura 3. Fecundidad absoluta (fec. abs.) de Leporinus obtusidens en la localidad de Ita Ibaté durante el lapso de estudio.
Tabla 3. Proporción de sexos en Leporinus obtusidens de ambos sitios y en todos los ciclos de muestreo.

\begin{tabular}{|c|c|c|c|c|c|c|c|}
\hline \multicolumn{8}{|c|}{ Ita Ibaté } \\
\hline ciclo & sexo & total & $\mathrm{M} / \mathrm{H}$ & Lmin & Lmax & Lprom & mes \\
\hline \multirow{4}{*}{ 04-05 } & $\mathrm{H}$ & 21 & \multirow{2}{*}{$3,3 / 1$} & 193 & 450 & 327 & oct \\
\hline & M & 71 & & 168 & 420 & 281 & ene - jun \\
\hline & $\mathrm{V}$ & 13 & \multirow{4}{*}{$1,6 / 1$} & 155 & 270 & 186 & jun \\
\hline & $\mathrm{H}$ & 48 & & 192 & 500 & 340 & sep-oct-nov \\
\hline \multirow[t]{2}{*}{ 05-06 } & M & 77 & & 180 & 422 & 293 & abr-oct-dic \\
\hline & $\mathrm{V}$ & 27 & & 132 & 290 & 210 & nov \\
\hline \multirow{3}{*}{ 06-07 } & $\mathrm{H}$ & 11 & \multirow{3}{*}{$5,09 / 1$} & 286 & 431 & 350 & sep \\
\hline & M & 56 & & 204 & 558 & 306 & sep \\
\hline & $\mathrm{V}$ & 5 & & 132 & 264 & 191 & s/dif \\
\hline \multirow{3}{*}{ 07-08 } & $\mathrm{H}$ & 22 & \multirow{2}{*}{$1,7 / 1$} & 215 & 450 & 356 & ago \\
\hline & M & 38 & & 137 & 370 & 284 & ago \\
\hline & $\mathrm{V}$ & 88 & \multirow{4}{*}{$2,8 / 1$} & 120 & 265 & 193 & ago \\
\hline \multirow{3}{*}{ 08-09 } & $\mathrm{H}$ & 13 & & 220 & 370 & 291 & $\mathrm{~s} /$ dif \\
\hline & M & 37 & & 190 & 358 & 281 & ago \\
\hline & $\mathrm{V}$ & 3 & & 184 & 230 & 213 & s/dif \\
\hline \multicolumn{8}{|c|}{ Ituzaingó } \\
\hline ciclo & sexo & total & $\mathrm{M} / \mathrm{H}$ & Lmin & Lmax & Lprom & mes \\
\hline \multirow{3}{*}{ 04-05 } & $\mathrm{H}$ & 30 & \multirow{3}{*}{$1 / 1,66$} & 166 & 485 & 330 & jul-sep-oct \\
\hline & M & 18 & & 142 & 412 & 294 & oct \\
\hline & V & 4 & & 144 & 272 & 214 & $\mathrm{~s} / \mathrm{dif}$ \\
\hline \multirow{2}{*}{$05-06$} & $\mathrm{H}$ & 33 & \multirow{3}{*}{$1 / 1,1$} & 200 & 452 & 340 & nov \\
\hline & M & 30 & & 179 & 410 & 294 & oct \\
\hline \multirow{4}{*}{$06-07$} & V & 18 & & 120 & 293 & 204 & $\mathrm{~s} / \mathrm{dif}$ \\
\hline & $\mathrm{H}$ & 31 & \multirow{3}{*}{$1 / 1,3$} & 170 & 467 & 353 & ene \\
\hline & M & 24 & & 245 & 510 & 329 & ene \\
\hline & V & 3 & & 250 & 298 & 276 & $\mathrm{~s} / \mathrm{dif}$ \\
\hline \multirow{3}{*}{ 07-08 } & $\mathrm{H}$ & 182 & \multirow{3}{*}{$1 / 2,2$} & 200 & 500 & 365 & jul-ago-nov \\
\hline & M & 82 & & 175 & 560 & 301 & ago-sep-nov \\
\hline & $\mathrm{V}$ & 106 & & 115 & 270 & 174 & jul-nov \\
\hline \multirow{3}{*}{ 08-09 } & $\mathrm{H}$ & 25 & \multirow{3}{*}{$1,4 / 1$} & 230 & 557 & 381 & oct \\
\hline & M & 36 & & 180 & 440 & 292 & oct \\
\hline & V & 7 & & 152 & 290 & 228 & $\mathrm{~s} / \mathrm{dif}$ \\
\hline
\end{tabular}

Total: total por sexo, M/H: relación macho/hembra, Lmin: largo mínimo, Lmax: largo máximo, Lprom: largo promedio, mes: período de mayor cantidad de individuos, H: hembra, M: macho, V: virginal.

en este caso es necesario profundizar el conocimiento sobre las fluctuaciones de la composición sexual de la población en el sitio control para encontrar la causa

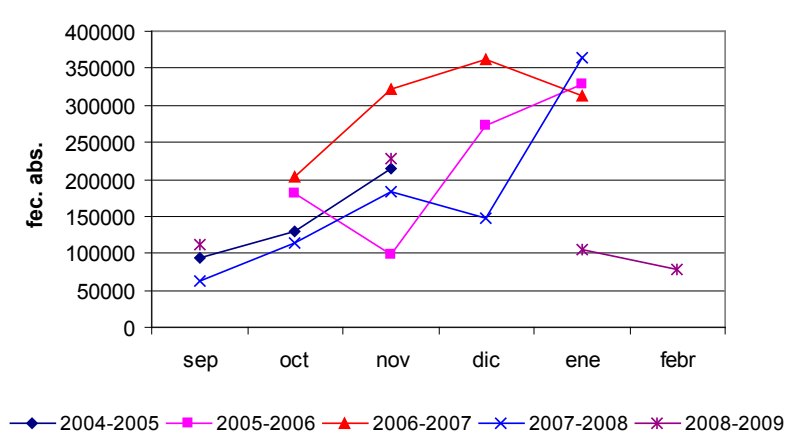

Figura 4. Fecundidad absoluta (fec. abs.) de Leporinus obtusidens en la localidad de Ituzaingó durante el lapso de estudio. 
del predominio de machos. La talla de las hembras fue equilibrada en ambos sitios, aunque si se consideran solo las hembras maduras éstas presentaron mayores tallas en Ituzaingó.

Según las características morfológicas, los ovarios fueron semejantes en ambos sitios estudiados, mostrando modificaciones intensas durante los meses de muestreo. Los cambios macroscópicos (aumento de tamaño y de la vascularización, cambios en la coloración y textura) fueron acompañados por grandes modificaciones histológicas durante el desarrollo de los ovocitos (formación de la zona radiata, envoltura folicular, membrana basal y teca), que fueron achatados y elongados en hembras inmaduras y voluminosos en el período de maduración avanzada. Microscópicamente, de la túnica de tejido conectivo que rodea los ovarios se desprenden las lamelas ovulígeras que están sustentando las células germinativas. Las lamelas presentaron distintos grados de desarrollo dependiendo de la fase del ciclo reproductivo en que se encontraba el animal. En ellas se observaron los ovocitos en distintas fases, rodeados de sus envoltorios celulares.

Los ovocitos en fase de ovogonia, células primordiales de la línea germinativa, estuvieron presentes

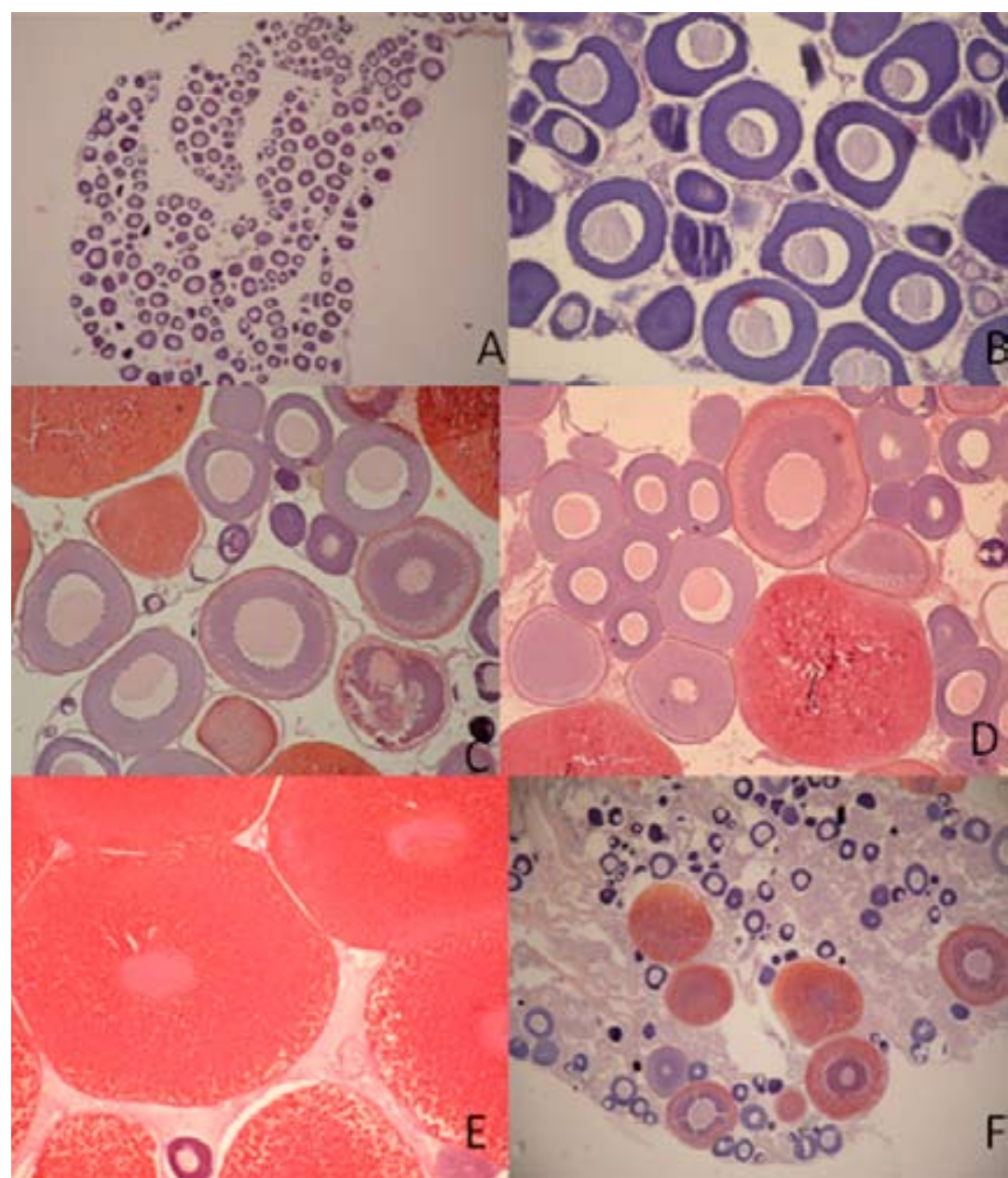

Figura 5. A y B: gónada en reposo, ovocitos de diferentes tamaños, citoplasma basófilo y nucléolos periféricos. C y D: gónada en inicio de maduración, ovocitos en fase III. E: gónada madura, ovocitos en fase IV. F: gónada desovada, restos de membranas plegadas. HE, 200X. como células redondas, basófilas y aisladas, siendo observados en todos los ciclos de muestreo (Figura 5A). Los ovocitos en fase I o cromatina-nucleolo se presentaron como células pequeñas, generalmente agrupadas en nidos, en número variado, habitualmente localizados en la periferia de las lamelas ovulígeras, con núcleo grande y central, ligeramente basófilo, nucleolo único (o poco numeroso) y fuertemente basófilo, relación núcleo citoplasma 1:1,5. Los ovocitos en fase II o perinucleolares fueron células de diferente tamaño de acuerdo al grado de maduración (Figura 5B), con núcleo grande, inicialmente oval y gradualmente más redondeado, con abundantes nucleolos próximos a la envoltura nuclear. El tamaño se incrementó acentuadamente en relación a la fase anterior y el contorno se tornó poliédrico. El citoplasma abundante, inicialmente filamentoso se observó fuertemente basófilo. A veces fue posible observar los cuerpos de Balbiani. En los ovocitos de mayor tamaño se detectó una capa de células foliculares.

Los ovocitos en fase III o alvéolo cortical se observaron como células mayores y más redondeadas que el estado anterior, presentaron núcleo voluminoso, con nucleolos basófilos junto a la membrana nuclear y nucleoplasma acidófilo, con citoplasma abundante, menos basófilo, presencia de alvéolos corticales en cantidades variables, próximos a la membrana plasmática (Figuras 5C y 5D). En los ovocitos más desarrollados, la capa folicular presentó en su superficie externa células achatadas que se correspondieron con las de la teca. Entre ésta y el ovocito, se destacó la denominada zona radiata o pelúcida, capa acelular, uniforme y acidófila.

Los ovocitos en fase IV o vitelogénicos fueron las células de mayor tamaño (Figura 5E). Presentaron el núcleo central, acidófilo, de contorno irregular, delimitado por gránulos de vitelo y presencia de nucleolos basófilos, citoplasma abundante, repleto de gránulos de vitelo. La zona radiata se visualizó con estrías radiales y la capa folicular y teca formada por células planas núcleo alargado y basófilo y núcleo poco definido. En los ovocitos con mayor desarrollo se observó el núcleo desplazado a la periferia (quiebra de vesícula germinativa) que ayudó a identificar el polo animal de la célula. Ovocitos atrésicos estaban presentes en las gónadas en todos los ciclos. Se visualizaron como células grandes, de citoplasma menos homogéneo, con pérdida 
de granulaciones citoplasmáticas y plegamiento de la membrana plasmática (Figura 5F).

Considerando todos los ciclos, la cantidad de ejemplares virginales fue equilibrada en ambos sitios. El ciclo 2007-2008 fue el período que se destacó por la abundancia de los ejemplares en este estadio de desarrollo. La RGS para este estadio de desarrollo osciló entre 0,03 a $0,75 \%$. Las hembras en reposo (Figura $5 \mathrm{~A}$ ) fueron capturadas con frecuencia en los dos lugares de muestreo. En los períodos 2007-2008 y 2008-2009 las hembras en reposo aparecieron en todos los meses de captura, en ambos sitios. La RGS para este estadio se situó entre 1,16 a 2,08\%. En reposo, los ovarios fueron traslúcidos, pequeños, con poca vascularización y moderado tejido conectivo. Se observó predominio de ovocitos en fase II con tamaño promedio de 169 micrómetros. El inicio de la maduración gonadal predominó en el mes de agosto en el sitio control y entre 1 y 2 meses más tarde en el sitio de impacto. Solo en el último ciclo se invirtió, observándose este estadio de desarrollo primero en Ituzaingó.

La RGS para el inicio de la maduración estuvo entre 5,60 y $6,35 \%$. Los ovarios aumentaron su tamaño, tuvieron vasos sanguíneos más evidentes y ovocitos visibles macroscópicamente. Microscópicamente presentaron predominio de ovocitos en fase III, con tamaño promedio de 360 micrómetros (Figura 5D). Desde el inicio de la primavera se capturaron ejemplares maduros en todos los años de muestreo. El ciclo 2008-2009 se caracterizó por tener este estadio hasta el mes de febrero inclusive. En este ciclo, en el sitio de impacto, la RGS duplicó la del sitio control. Los valores en los distintos períodos oscilaron entre 15 y $22 \%$. Las hembras en este estadio presentaron ovarios grandes, ocupando toda la cavidad abdominal; el color varió de gris verdoso a verde oliva y las gónadas mostraron importante vascularización. Las lamelas estaban ocupadas por ovocitos en fase IV, sin embargo se encontraron cantidades menores de ovocitos en fases II y III.

El tamaño promedio de los ovocitos en fase IV fue de 630 micrómetros, ubicándose la boga dentro de la gran mayoría de peces que tienen ovocitos maduros con diámetros superiores a 500 micrómetros ${ }^{18}$. Para algunos autores es difícil establecer una relación entre el diámetro de ovocitos y la longitud del animal. A su vez, aunque es aceptado que huevos grandes producen larvas más grandes, el criterio del tamaño del huevo para evaluar la calidad del mismo, continúa generando gran controversia. En truchas se indica que el tamaño del huevo no tendría importancia sobre su calidad ${ }^{7,8}$. No obstante, algunos autores señalan que la variación en el diámetro del huevo parece ser uno de los criterios mas importante en la calidad del huevo para peces ${ }^{9,10}$.

La variabilidad intraespecífica del tamaño del huevo esta asociado con la edad, el tamaño y las condiciones fisiológicas de la hembra, con el tiempo de desove y la variación en las condiciones medioambientales en que se encuentra el pez ${ }^{3}$. En el sitio control se observó una ligera diferencia en el tamaño de los ovocitos que superó las 700 micrómetros en el ciclo de bajos niveles hidrométricos (2004-2005), posiblemente por la ligera prolongación del tiempo de permanencia de los ovocitos en la cavidad celómica causada por la falta de estímulos suficientes para el desove.

El ciclo donde se concentraron nítidamente los animales capturados en posdesove fue 2008-2009. Marzo fue el mes en común en todos los ciclos de muestreo donde se registraron hembras desovadas. La RGS declinó bruscamente hasta valores de 3,3 a $4,14 \%$. La figura microscópica que caracterizó al estadio fue la presencia de ovocitos atrésicos que estaban presentes en las gónadas en todos los ciclos. En algunas especies se reportó incidencia de atresia superior al $37 \%{ }^{16}$. Sin embargo varios autores destacan que si bien los niveles de atresia varían con el estadio del ciclo reproductivo, este es un proceso poco común en peces bajo óptimas condiciones ${ }^{17}$. El proceso no jugaría un rol en el control de la fecundidad en ovarios normales y la frecuencia aumenta en relación a las condiciones ambientales estresantes. En este trabajo se detectaron como células predominantes exclusivamente en el estadio de posdesove, no registrándose diferencias entre ciclos ni sitios.

En conclusión, pese a que las represas modifican numerosas características de un río, obligando a todo el ecosistema a una reacomodación para alcanzar el equilibrio, proceso durante el cual muchas especies tienden a desaparecer, especialmente las poblaciones migradoras, los resultados de este trabajo sugieren que el sistema en cercanías de la represa Yaciretá presenta condiciones favorables para Leporinus obtusidens.

Agradecimientos. A la Entidad Binacional Yacyretá (EBY), quien proporcionó el financiamiento necesario para la realización de este trabajo. Al personal del Área de Medio Ambiente de la EBY en Ituzaingó (Corrientes, Argentina), que brindó apoyo técnico y logístico para los muestreos.

\section{REFERENCIAS}

1. Agostinho A. 1995. Consideraçoes sobre atuação do sêtor elétrico na preservação da fauna aquática e dos recursos pesqueiros. Anais Seminário sobre fauna aquática e o setor elétrico brasileiro. Publ. Electrobrás-Comase, Río de Janeiro, Caderno 4: 8-19.

2. Barbieri G, Pereira E. 1988. Análise comparativa do crescimento e de aspectos reprodutivos da piava, Leporinus friderici (Blach, 1794) (Osteichthyes, Anostomidae) da represa do lobo e do rio Mogi-Guaçu, Estado do Sao Paulo. Ciência e Cultura (Campinas, Brasil) 40: 693-697.

3. Baynes S, Howell B. 1996. The influence of egg size and incubation temperature on the condition of Solea solea (L.) larvae at hatching and first feeding. J Exp Mar Biol 199: 59-77.

4. Bechara J, Roux J, Terraes J, Sánchez S, Toccalino P, González A, Ortiz J. 2000. Evaluación de los recursos pesqueros aguas abajo de la represa. Informe Final, con- 
venio EBY-UNNE Corrientes (Argentina), Acta Complem. $\mathrm{N}^{\mathrm{o}} 7,158 \mathrm{p}$.

5. Bechara J, Ruiz Díaz F. 2004. Effects of mean annual discharge an dam regulation on fish biomas in the high Paraná river (Argentina). Proceedings of the Fifth International Symposium on Ecohydraulics, Madrid, p. 95-101.

6. Bechara J. 2005. Evaluación de los recursos pesqueros aguas abajo de la represa. Informe Final, convenio EBYUNNE Corrientes (Argentina), Acta Complem. No 16, 146 p.

7. Bromage N, Cumaranatunga P. 1988. Egg production in the rainbow trout. In: Recent advances in aquaculture (Muir JF, Roberts RJ Ed.), Timber Press, London, p. 63138.

8. Bromage N, Jones J, Randall C, Trush M, Davies B, Springate J, Duston J, Barker G. 1991. Broodstock management, fecundity, egg quality and the timing of production in the rainbow trout (Oncorhynchuss mykiss). Aquaculture 100: 141-166.

9. Bromage N. 1995. Broodstock management and seed quality general considerations. In: Broodstock management and egg and larval quality (Bromage $\mathrm{N}$, Roberts RJ Ed.), Blackwell Science, Oxford, p. 1-24.

10. Kjorsvik E, Mangor A, Holmefjord I. 1990. Egg quality in fishes. Adv Mar Biol 26: 71-113.

11. Meichtry N. 1999. La comunidad fitoplanctónica durante las primeras etapas de llenado del embalse de Yacyretá, Argentina. Rev Ictiol (Argentina) 7: 15-26.
12. Pankhurst N, Van Der Kraak G. 2000. Evidence that acute stress inhibits ovarian steroidogenesis in rainbow trout in vivo, through the action of cortisol. Gen \& Comp Endocr 117: 225-237.

13. Romagosa E. 1988. Desenvolvimento gonadal (morfología; ultra-estrutura) e indução da reprodução do matrinxá, Brycon cephalus (Günther, 1869) em cautiverio. Tese Doutorado, Universidade Federal de Sao Carlos (Brasil), 218 p.

14. Romagosa E. 1991. Mudanças morfológicas (microscopia de luz e eletronica) das gónadas do pacú Piaractus mesopotamicus durante o ciclo reproductivo e em condições de confinamento. Dissertação Maestrado em Ciencias, Instituto de Biociencias, Universidad Estadual Paulista, Rio Claro (Brasil), $177 \mathrm{p}$.

15. Schreck C, Contreras W, Fizpatrick M. 2001. Effects of stress on fish reproduction, gamete quality, and progeny. Aquaculture 197: 3-24.

16. Tam W, Roy R, Makaran R. 1986. Ovarian cycle and plasma concentration of oestrogen and vitellogenin in the brook trout (Salvelinus fontinalis, Mitchell). Can J Zool 64: 744-751.

17. Tyler C, Sumpter J. 1996. Oocite growth and development in teleosts. Rev Fish Biol \& Fisheries 6: 287-318.

18. Vazzoler AE. 1996. Biología da reprodução de peixes teleosteos: teoria e prática, Publ. Universidade Estadual de Maringá, Maringá (Brasil), 169 p. 